\title{
Review of: "Polymer microparticles prolong delivery of the 15-PGDH inhibitor SW033291"
}

\author{
André Luís Morais Ruela
}

Potential competing interests: The author(s) declared that no potential competing interests exist.

This work was clearly designed and executed. the results are encouraging, demonstrating the feasibility of an injectable formulation ( $\beta-C D$ carriers loaded with (+)SW033291) in the treatment of chronic fibrosis.

Drug targeting to the lungs was possibly achieved. I am strongly in accordance with the publication of this paper. However, some brief discussions concerning issues like cytotoxicity of $\beta-C D$ carriers loaded with (+)SW033291 and its biodegradability must be encouraged. 